\title{
New upper bound for a class of vertex Folkman numbers
}

\author{
N. Kolev \\ Department of Algebra \\ Faculty of Mathematics and Informatics \\ "St. Kl. Ohridski" University of Sofia \\ 5 J. Bourchier blvd, 1164 Sofia \\ BULGARIA \\ N. Nenov \\ Department of Algebra \\ Faculty of Mathematics and Informatics \\ "St. Kl. Ohridski" University of Sofia \\ 5 J. Bourchier blvd, 1164 Sofia \\ BULGARIA \\ nenov@fmi.uni-sofia.bg
}

Submitted: Jun 9, 2005; Accepted: Feb 7, 2006; Published: Feb 15, 2006

Mathematics Subject Classification: 05C55

\begin{abstract}
Let $a_{1}, \ldots, a_{r}$ be positive integers, $m=\sum_{i=1}^{r}\left(a_{i}-1\right)+1$ and $p=\max \left\{a_{1}, \ldots, a_{r}\right\}$. For a graph $G$ the symbol $G \rightarrow\left\{a_{1}, \ldots, a_{r}\right\}$ denotes that in every $r$-coloring of the vertices of $G$ there exists a monochromatic $a_{i}$-clique of color $i$ for some $i=1, \ldots, r$. The vertex Folkman numbers $F\left(a_{1}, \ldots, a_{r} ; m-1\right)=\min \left\{|V(G)|: G \rightarrow\left(a_{1} \ldots a_{r}\right)\right.$ and $K_{m-1} \nsubseteq \subseteq G$ are considered. We prove that $F\left(a_{1}, \ldots, a_{r} ; m-1\right) \leq m+3 p$, $p \geq 3$. This inequality improves the bound for these numbers obtained by Łuczak, Ruciński and Urbański (2001).
\end{abstract}

\section{Introduction}

We consider only finite, non-oriented graphs without loops and multiple edges. We call a $p$-clique of the graph $G$ a set of $p$ vertices, each two of which are adjacent. The largest positive integer $p$, such that the graph $G$ contains a $p$-clique is denoted by $c l(G)$. In this paper we shall also use the following notations:

$V(G)$ - vertex set of the graph $G$;

$E(G)$ - edge set of the graph $G$; 
$\bar{G}$ - the complement of $G$;

$G[V], V \subseteq V(G)$ - the subgraph of $G$ induced by $V$;

$G-V$ - the subgraph induced by the set $V(G) \backslash V$;

$N_{G}(v), v \in V(G)$ - the set of all vertices of $G$ adjacent to $v$;

$K_{n}$ - the complete graph on $n$ vertices;

$C_{n}$ - simple cycle on $n$ vertices;

$P_{n}$ - path on $n$ vertices;

$\chi(G)$ - the chromatic number of $G$;

$\lceil x\rceil$ - the least positive integer greater or equal to $x$.

Let $G_{1}$ and $G_{2}$ be two graphs without common vertices. We denote by $G_{1}+G_{2}$ the graph $G$ for which $V(G)=V\left(G_{1}\right) \cup V\left(G_{2}\right)$ and $E(G)=E\left(G_{1}\right) \cup E\left(G_{2}\right) \cup E^{\prime}$, where $E^{\prime}=\left\{[x, y] \mid x \in V\left(G_{1}\right), y \in V\left(G_{2}\right)\right\}$.

Definition Let $a_{1}, \ldots, a_{r}$ be positive integers. We say that the $r$-coloring

$$
V(G)=V_{1} \cup \ldots \cup V_{r}, V_{i} \cap V_{j}=\emptyset, i \neq j,
$$

of the vertices of the graph $G$ is $\left(a_{1}, \ldots, a_{r}\right)$-free, if $V_{i}$ does not contain an $a_{i}$-clique for each $i \in\{1, \ldots, r\}$. The symbol $G \rightarrow\left(a_{1}, \ldots, a_{r}\right)$ means that there is no $\left(a_{1}, \ldots, a_{r}\right)$-free coloring of the vertices of $G$.

We consider for arbitrary natural numbers $a_{1}, \ldots, a_{r}$ and $q$

$$
H\left(a_{1}, \ldots a_{r} ; q\right)=\left\{G: G \rightarrow\left(a_{1}, \ldots, a_{r}\right) \text { and } c l(G)<q\right\} .
$$

The vertex Folkman numbers are defined by the equalities

$$
F\left(a_{1}, \ldots, a_{r} ; q\right)=\min \left\{|V(G)|: G \in H\left(a_{1}, \ldots, a_{r} ; q\right)\right\} .
$$

It is clear that $G \rightarrow\left(a_{1}, \ldots, a_{r}\right)$ implies $\operatorname{cl}(G) \geq \max \left\{a_{1}, \ldots, a_{r}\right\}$. Folkman [3] proved that there exists a graph $G$ such that $G \rightarrow\left(a_{1}, \ldots, a_{r}\right)$ and $c l(G)=\max \left\{a_{1}, \ldots, a_{r}\right\}$. Therefore

$$
F\left(a_{1}, \ldots, a_{r} ; q\right) \text { exists if and only if } q>\max \left\{a_{1}, \ldots, a_{r}\right\} .
$$

These numbers are called vertex Folkman numbers. In [5] Łuczak and Urbański defined for arbitrary positive integers $a_{1}, \ldots, a_{r}$ the numbers

$$
m=m\left(a_{1}, \ldots, a_{r}\right)=\sum_{i=1}^{r}\left(a_{i}-1\right)+1 \text { and } p=p\left(a_{1}, \ldots, a_{r}\right)=\max \left\{a_{1}, \ldots, a_{r}\right\} .
$$

Obviously $K_{m} \rightarrow\left(a_{1}, \ldots, a_{r}\right)$ and $K_{m-1} \nrightarrow\left(a_{1}, \ldots, a_{r}\right)$. Therefore if $q \geq m+1$ then $F\left(a_{1}, \ldots, a_{r} ; q\right)=m$.

From (1) it follows that the number $F\left(a_{1}, \ldots, a_{r} ; q\right)$ exists if and only if $q \geq p+1$. Luczak and Urbański [5] proved that $F\left(a_{1}, \ldots, a_{r} ; m\right)=m+p$. Later, in [6], Luczak, Ruciński and Urbański proved that $K_{m-p-1}+\bar{C}_{2 p+1}$ is the only graph in $H\left(a_{1}, \ldots, a_{r} ; m\right)$ with $m+p$ vertices. 
From (1) it follows that the number $F\left(a_{1}, \ldots, a_{r} ; m-1\right)$ exists if and only if $m \geq p+2$. An overview of the results about the numbers $F\left(a_{1}, \ldots, a_{r} ; m-1\right)$ was given in [1]. Here we shall note only the general bounds for the numbers $F\left(a_{1}, \ldots, a_{r} ; m-1\right)$. In [8] the following lower bound was proved

$$
F\left(a_{1}, \ldots, a_{r} ; m-1\right) \geq m+p+2, p \geq 2 .
$$

In the above inequality an equality occurs in the case when $\max \left\{a_{1}, \ldots, a_{r}\right\}=2$ and $m \geq 5$ (see [4],[6],[7]). For these reasons we shall further consider only the numbers $F\left(a_{1}, \ldots, a_{r} ; m-1\right)$ when $\max \left\{a_{1}, \ldots, a_{r}\right\} \geq 3$.

In [6] Łuczak, Ruciński and Urbański proved the following upper bound for the numbers $F\left(a_{1}, \ldots, a_{r} ; m-1\right)$ :

$$
F\left(a_{1}, \ldots, a_{r} ; m-1\right) \leq m+p^{2}, \text { for } m \geq 2 p+2 .
$$

In [6] they also announced without proof the following inequality:

$$
F\left(a_{1}, \ldots, a_{r} ; m-1\right) \leq 3 p^{2}+p-m p+2 m-3, \text { for } p+3 \leq m \leq 2 p+1 .
$$

In this paper we shall improve these bounds proving the following

Main theorem Let $a_{1}, \ldots, a_{r}$ be positive integers and $m$ and $p$ be defined by (2). Let $m \geq p+2$ and $p \geq 3$. Then

$$
F\left(a_{1}, \ldots, a_{r} ; m-1\right) \leq m+3 p .
$$

Remark This bound is exact for the numbers $F(2,2,3 ; 4)$ and $F(3,3 ; 4)$ because

$$
F(2,2,3 ; 4)=14(\text { see }[2]) \text { and } F(3,3 ; 4)=14(\text { see }[9]) .
$$

\section{Main construction}

We consider the cycle $C_{2 p+1}$. We assume that

$$
V\left(C_{2 p+1}\right)=\left\{v_{1}, \ldots, v_{2 p+1}\right\}
$$

and

$$
E\left(C_{2 p+1}\right)=\left\{\left[v_{i}, v_{i+1}\right], i=1, \ldots, 2 p\right\} \cup\left\{v_{1}, v_{2 p+1}\right\} .
$$

Let $\sigma$ denote the cyclic automorphism of $C_{2 p+1}$, i.e. $\sigma\left(v_{i}\right)=v_{i+1}$ for $i=1, \ldots, 2 p$, $\sigma\left(v_{2 p+1}\right)=v_{1}$. Using this automorphism and the set $M_{1}=V\left(C_{2 p+1}\right) \backslash\left\{v_{1}, v_{2 p-1}, v_{2 p-2}\right\}$ we define $M_{i}=\sigma^{i-1}\left(M_{1}\right)$ for $i=1, \ldots, 2 p+1$. Let $\Gamma_{p}$ denote the extension of the graph $\bar{C}_{2 p+1}$ obtained by adding the new pairwise independent vertices $u_{1}, \ldots, u_{2 p+1}$ such that

$$
N_{\Gamma_{p}}\left(u_{i}\right)=M_{i} \text { for } i=1, \ldots, 2 p+1 .
$$


We easily see that $c l\left(\bar{C}_{2 p+1}\right)=p$.

Now we extend $\sigma$ to an automorphism of $\Gamma_{p}$ via the equalities $\sigma\left(u_{i}\right)=u_{i+1}$, for $i=1, \ldots, 2 p$, and $\sigma\left(u_{2 p+1}\right)=u_{1}$. Now it is clear that

$$
\sigma \text { is an automorphism of } \Gamma_{p} .
$$

The graph $\Gamma_{p}$ was defined for the first time in [8]. In [8] it is also proved that $\Gamma_{p} \rightarrow(3, p)$ for $p \geq 3$. For the proof of the main theorem we shall also use the following generalisation of this fact.

Theorem 1 Let $p \geq 3$ be a positive integer and $m=p+2$. Then for arbitrary positive integers $a_{1}, \ldots, a_{r}$ ( $r$ is not fixed) such that

$$
m=1+\sum_{i=1}^{r}\left(a_{i}-1\right)
$$

and $\max \left\{a_{1}, \ldots, a_{r}\right\} \leq p$ we have

$$
\Gamma_{p} \rightarrow\left(a_{1}, \ldots a_{r}\right)
$$

\section{$3 \quad$ Auxiliary results}

The next proposition is well known and easy to prove.

Proposition 1 Let $a_{1}, \ldots, a_{r}$ be positive integers and $n=a_{1}+\ldots+a_{r}$. Then

$$
\left\lceil\frac{a_{1}}{2}\right\rceil+\ldots+\left\lceil\frac{a_{r}}{2}\right\rceil \geq\left\lceil\frac{n}{2}\right\rceil .
$$

If $n$ is even than this inequality is strict unless all the numbers $a_{1}, \ldots, a_{r}$ are even. If $n$ is odd then this inequality is strict unless exactly one of the numbers $a_{1}, \ldots, a_{r}$ is odd.

Let $P_{k}$ be the simple path on $k$ vertices. Let us assume that

$$
V\left(P_{k}\right)=\left\{v_{1}, \ldots, v_{k}\right\}
$$

and

$$
E\left(P_{k}\right)=\left\{\left[v_{i}, v_{i+1}\right], i=1, \ldots, k-1\right\} .
$$

We shall need the following obvious facts for the complementary graph $\bar{P}_{k}$ of the graph $P_{k}$ :

$$
\begin{gathered}
\operatorname{cl}\left(\bar{P}_{k}\right)=\left\lceil\frac{k}{2}\right\rceil \\
\operatorname{cl}\left(\bar{P}_{2 k}-v\right)=\operatorname{cl}\left(\bar{P}_{2 k}\right), \text { for each } v \in V\left(\bar{P}_{2 k}\right) \\
\operatorname{cl}\left(\bar{P}_{2 k}-\left\{v_{2 k-2}, v_{2 k-1}\right\}\right)=\operatorname{cl}\left(\bar{P}_{2 k}\right) \text { for } k \geq 2 \\
c l\left(\bar{P}_{2 k+1}-v_{2 i}\right)=\operatorname{cl}\left(\bar{P}_{2 k+1}\right), i=1, \ldots, k, k \geq 1 .
\end{gathered}
$$

The proof of Theorem 1 is based upon three lemmas. 
Lemma 1 Let $V \subset V\left(C_{2 p+1}\right)$ and $|V|=n<2 p+1$. Let $G=\bar{C}_{2 p+1}[V]$ and let $G_{1}, \ldots, G_{s}$ be the connected components of the graph $\bar{G}=C_{2 p+1}[V]$. Then

$$
c l(G) \geq\left\lceil\frac{n}{2}\right\rceil .
$$

If $n$ is even, then (9) is strict unless all $\left|V\left(G_{i}\right)\right|$ for $i=1, \ldots, s$ are even. If $n$ is odd, then (9) is strict unless exactly one of the numbers $\left|V\left(G_{i}\right)\right|$ is odd.

Proof Let us observe that

$$
G=\bar{G}_{1}+\ldots+\bar{G}_{s}
$$

Since $V \neq V\left(C_{2 p+1}\right)$ each of the graphs $G_{i}$ is a path. From (10) and (5) it follows that

$$
c l(G)=\sum_{i=1}^{s}\left\lceil\frac{n_{i}}{2}\right\rceil,
$$

where $n_{i}=\left|V\left(G_{i}\right)\right|, i=1, \ldots, s$. From this inequality and Proposition 1 we obtain the inequality (9). From Proposition 1 it also follows that if $n$ is even then there is equality in (9) if and only if the numbers $n_{1}, \ldots, n_{s}$ are even, and if $n$ is odd then we have equality in (9) if and only if exactly one of the numbers $n_{1}, \ldots, n_{s}$ is odd.

Corollary 1 It is true that $\operatorname{cl}\left(\Gamma_{p}\right)=p$.

Proof It is obvious that $\operatorname{cl}\left(\bar{C}_{2 p+1}\right)=p$ and hence $c l\left(\Gamma_{p}\right) \geq p$. Let us denote an arbitrary maximal clique of $\Gamma_{p}$ by $Q$. Let us assume that $|Q|>p$. Then $Q$ must contain a vertex $u_{i}$ for some $i=1, \ldots, 2 p+1$. As the vertices $u_{i}$ are pairwise independent $Q$ must contain at most one of them. Since $\sigma$ is an automorphism of $\Gamma_{p}$ (see (4)) and $u_{i}=\sigma^{i-1}\left(u_{1}\right)$, we may assume that $Q$ contains $u_{1}$. Let us assign the subgraph of $\Gamma_{p}$ induced by $N_{\Gamma_{p}\left(u_{1}\right)}=M_{1}$ by $H$. The connected components of $H$ are $\left\{v_{2}, v_{3}, \ldots, v_{2 p-3}\right\}$ and $\left\{v_{2 p}, v_{2 p+1}\right\}$ and both of them contain an even number of vertices. Using Lemma 1 we have $c l(H)=p-1$. Hence $|Q|=p$ and this contradicts the assumption.

The next two lemmas follow directly from (10), (6), (7), and (8) and need no proof.

Lemma 2 Let $V \subsetneq V\left(C_{2 p+1}\right)$ and $G=\bar{C}_{2 p+1}[V]$. Let $P_{k}=\left\{v_{1}, v_{2}, \ldots, v_{k}\right\}$ be a connected component of the graph $\bar{G}=C_{2 p+1}[V]$. Then

(a) if $k=2 s$ then

$$
c l\left(G-v_{i}\right)=\operatorname{cl}(G), i=1, \ldots, 2 s,
$$

and

$$
c l\left(G-\left\{v_{2 s-2}, v_{2 s-1}\right\}\right)=\operatorname{cl}(G) .
$$

(b) if $k=2 s+1$ then

$$
c l\left(G-v_{2 i}\right)=c l(G), i=1, \ldots, s .
$$


Lemma 3 Let $V \subseteq V\left(C_{2 p+1}\right)$ and $\bar{C}_{2 p+1}=G$. Let

$$
P_{2 k}=\left\{v_{1}, \ldots, v_{2 k}\right\} \quad \text { and } P_{s}=\left\{w_{1}, \ldots, w_{s}\right\}
$$

be two connected components of the graph $\bar{G}=C_{2 p+1}[V]$. Then

(a) if $s=2 t$ then

$$
c l\left(G-\left\{v_{i}, w_{j}\right\}\right)=\operatorname{cl}(G),
$$

for $i=1, \ldots, 2 k, j=1, \ldots, s$, and

$$
c l\left(G-\left\{v_{2 k-2}, v_{2 k-1}, w_{j}\right\}\right)=\operatorname{cl}(G),
$$

for $j=1, \ldots, s$.

(b) If $s=2 t+1$ then

$$
c l\left(G-\left\{v_{2 k-2}, v_{2 k-1}, w_{2 i}\right\}\right)=\operatorname{cl}(G), \text { for } i=1, \ldots, t .
$$

\section{Proof of Theorem 1}

We shall prove Theorem 1 by induction on $r$. As $m=\sum_{i=1}^{r}\left(a_{i}-1\right)+1=p+2$ and $\max \left\{a_{1}, \ldots, a_{r}\right\} \leq p$ we have $r \geq 2$. Therefore the base of the induction is $r=2$. We warn the reader that the proof of the inductive base is much more involved then the proof of the inductive step. Let $r=2$ and $\left(a_{1}-1\right)+\left(a_{2}-1\right)+1=p+2$ and $\max \left\{a_{1}, a_{2}\right\} \leq p$. Then we have

$$
a_{1}+a_{2}=p+3 .
$$

Since $p \geq 3$ and $\max \left\{a_{1}, a_{2}\right\} \leq p$ we have that

$$
a_{i} \geq 3, i=1,2 \text {. }
$$

We must prove that $\Gamma_{p} \rightarrow\left(a_{1}, a_{2}\right)$. Assume the opposite and let $V\left(\Gamma_{p}\right)=V_{1} \cup V_{2}$ be a $\left(a_{1}, a_{2}\right)$-free coloring of $V\left(\Gamma_{p}\right)$. Define the sets

$$
V_{i}^{\prime}=V_{i} \cap V\left(\bar{C}_{2 p+1}\right), i=1,2,
$$

and the graphs

$$
G_{i}=\bar{C}_{2 p+1}\left[V_{i}^{\prime}\right], i=1,2 .
$$

By assumption $\Gamma_{p}\left[V_{i}\right]$ does not contain an $a_{i}$-clique and hence $\Gamma_{p}\left[V_{i}^{\prime}\right]$ does not contain an $a_{i}$-clique, too. Therefore from Lemma 1 we have $\left|V_{i}^{\prime}\right| \leq 2 a_{i}-2, i=1,2$. From these inequalities and the equality

$$
\left|V_{1}^{\prime}\right|+\left|V_{2}^{\prime}\right|=2 p+1=2 a_{1}+2 a_{2}-5
$$

(as $p=a_{1}+a_{2}-3$, see (11)) we have two possibilities:

$$
\left|V_{1}^{\prime}\right|=2 a_{1}-2,\left|V_{2}^{\prime}\right|=2 a_{2}-3
$$


or

$$
\left|V_{1}^{\prime}\right|=2 a_{1}-3,\left|V_{2}^{\prime}\right|=2 a_{2}-2 .
$$

Without loss of generality we assume that

$$
\left|V_{1}^{\prime}\right|=2 a_{1}-2,\left|V_{2}^{\prime}\right|=2 a_{2}-3 .
$$

From (13) and Lemma 1 we obtain $c l\left(G_{i}\right) \geq a_{i}-1$ and by the assumption that the coloring $V_{1} \cup V_{2}$ is $\left(a_{1}, a_{2}\right)$-free we have

$$
\operatorname{cl}\left(G_{i}\right)=a_{i}-1 \text { for } i=1,2 .
$$

From (13), (14) and Lemma 1 we conclude that

The number of the vertices of each connected component of $\bar{G}_{1}$ is an even number;

and

the number of the vertices of exactly one of the connected components of $\bar{G}_{2}$ is an odd number.

According to (15) there are two possible cases.

Case 1. Some connected component of $\bar{G}_{1}$ has more then two vertices. Now from (15) it follows that this component has at least four vertices. Taking into consideration (15) and (4) we may assume that $\left\{v_{1}, \ldots, v_{2 s}\right\}, s \geq 2$, is a connected component of $\bar{G}_{1}$. Since $V_{1}^{\prime}$ does not contain an $a_{1}$-clique we have by Lemma 1 that $s<a_{1}$. Therefore $2 s+2 \leq 2 p$ and we can consider the vertex $u_{2 s+2}$.

Subcase 1.a. Assume that $u_{2 s+2} \in V_{1}$. Let $v_{2 s+2} \in V_{2}^{\prime}$. We have from (3) that

$$
N_{\Gamma_{p}}\left(u_{2 s+2}\right) \supseteq V_{1}^{\prime}-\left\{v_{2 s-2}, v_{2 s-1}\right\} .
$$

From (14) and Lemma 2(a) we have that the subgraph induced by $V_{1}^{\prime}-\left\{v_{2 s-2}, v_{2 s-1}\right\}$ contains an $\left(a_{1}-1\right)$-clique $Q$. From (17) it follows that $Q \cup\left\{u_{2 s+2}\right\}$ is an $a_{1}$-clique in $V_{1}$ which is a contradiction.

Now let $v_{2 s+2} \in V_{1}^{\prime}$. From (3) we have

$$
N_{\Gamma_{P}}\left(u_{2 s+2}\right) \supseteq V_{1}^{\prime}-\left\{v_{2 s-2}, v_{2 s-1}, v_{2 s+2}\right\} .
$$

According to (15) we can apply Lemma 3(a) for the connected component $\left\{v_{1}, \ldots, v_{2 s}\right\}$ of $\bar{G}_{1}$ and the connected component of $\bar{G}_{1}$ that contains $v_{2 s+2}$. We see from (14) and Lemma $3(\mathrm{a})$ that $V_{1}^{\prime}-\left\{v_{2 s-2}, v_{2 s-1}, v_{2 s+2}\right\}$ contains an $\left(a_{1}-1\right)$-clique $Q$ of the graph $G_{1}$. Now from (18) it follows that $Q \cup\left\{u_{2 s+2}\right\}$ is an $a_{1}$-clique in $V_{1}$, which is a contradiction.

Subcase 1.b. Assume that $u_{2 s+2} \in V_{2}$. If $v_{2 s+2} \notin V_{2}^{\prime}$ then from (3) it follows

$$
N_{\Gamma_{p}}\left(u_{2 s+2}\right) \supseteq V_{2}^{\prime}
$$


As $V_{2}^{\prime}$ contains an $\left(a_{2}-1\right)$-clique Q (see (14)). From (19) it follows that $Q \cup\left\{u_{2 s+2}\right\}$ is an $a_{2}$-clique in $V_{2}$, which is a contradiction.

Now let $v_{2 s+2} \in V_{2}^{\prime}$. In this situation we have from (3)

$$
N_{\Gamma_{p}}\left(u_{2 s+2}\right) \supseteq V_{2}^{\prime}-\left\{v_{2 s+2}\right\} .
$$

We shall prove that

$$
V_{2}-\left\{v_{2 s+2}\right\} \text { contains an }\left(a_{2}-1\right) \text {-clique of } \Gamma_{p} \text {. }
$$

As $v_{2 s}$ is the last vertex in the connected component of $G_{1}$, we have $v_{2 s+1} \in V_{2}^{\prime}$. Let $L$ be the connected component of $\bar{G}_{2}$ containing $v_{2 s+2}$. Now we have $L=\left\{v_{2 s+1}, v_{2 s+2}, \ldots\right\}$. Now (21) follows from Lemma 2 applied to the component $L$. From (20) and(21) it follows that $V_{2}$ contains an $a_{2}$-clique, which is a contradiction.

Case 2. Let all connected components of $\bar{G}_{1}$ have exactly two vertices.

From (12) and (13) it follows that $\bar{G}_{1}$ has at least two connected components. It is clear that $\bar{G}_{2}$ also has at least two components. From (16) we have that the number of the vertices of at least one of the components of $G_{2}$ is even. From these considerations and (4) it follows that it is enough to consider the situation when $\left\{v_{1}, v_{2}\right\}$ is a connected component of $\bar{G}_{1}$ and $\left\{v_{3}, \ldots, v_{2 s}\right\}$ is a component of $\bar{G}_{2}$, and $\left\{v_{2 s+1}, v_{2 s+2}\right\}$ is a component of $\bar{G}_{1}$. We shall consider two subcases.

Subcase 2.a. If $u_{2 s+2} \in V_{1}$.

Let $s=2$. We apply Lemma $3\left(\right.$ a) to the components $\left\{v_{1}, v_{2}\right\}$ and $\left\{v_{5}, v_{6}\right\}$. From (14) we conclude that

$$
V_{1}^{\prime}-\left\{v_{2}, v_{6}\right\} \text { contains an }\left(a_{1}-1\right) \text {-clique. }
$$

From (3) we have

$$
N_{\Gamma_{p}}\left(u_{6}\right) \supseteq V_{1}^{\prime}-\left\{v_{2}, v_{6}\right\} .
$$

Now (22) and (23) give that $V_{1}$ contains an $a_{1}$-clique.

Let $s \geq 3$. From (3) we have

$$
N_{\Gamma_{p}}\left(u_{2 s+2}\right) \supseteq V_{1}^{\prime}-\left\{v_{2 s+2}\right\} .
$$

According to Lemma 2(a) $V_{1}^{\prime}-\left\{v_{2 s+2}\right\}$ contains an $\left(a_{1}-1\right)$-clique. Now using (24) we have that this $\left(a_{1}-1\right)$-clique together with the vertex $u_{2 s+2}$ gives an $a_{1}$-clique in $V_{1}$. Subcase 2.a. is proved.

Subcase 2.b. Let $u_{2 s+2} \in V_{2}$.

Let $s=2$. From (3) we have $N_{\Gamma_{p}}\left(u_{6}\right) \supseteq V_{2}^{\prime}-\left\{v_{3}\right\}$. According to Lemma 2(a) and (14) $V_{2}^{\prime}-\left\{v_{3}\right\}$ contains an $\left(a_{2}-1\right)$-clique. This clique together with $u_{2 s+2} \in V_{2}$ gives an $a_{2}$-clique in $V_{2}$, which is a contradiction.

Let $s \geq 3$. Here from (3) we have $N_{\Gamma_{p}}\left(u_{2 s+2}\right) \supseteq V_{2}^{\prime}-\left\{v_{2 s-2}, v_{2 s-1}\right\}$. According to Lemma 2(a) and (14) we have that $V_{2}^{\prime}-\left\{v_{2 s-2}, v_{2 s-1}\right\}$ contains an $\left(a_{2}-1\right)$-clique. This 
clique together with $u_{2 s+2} \in V_{2}$ gives an $a_{2}$-clique in $V_{2}$, which is a contradiction. This completes the proof of case 2 and of the inductive base $r=2$.

Now we more easily handle the case $r \geq 3$. It is clear that

$$
G \rightarrow\left(a_{1}, \ldots, a_{r}\right) \Leftrightarrow G \rightarrow\left(a_{\varphi(1)}, \ldots, a_{\varphi(r)}\right)
$$

for any permutation $\varphi \in S_{r}$. That is why we may assume that

$$
a_{1} \leq \ldots \leq a_{r} \leq p
$$

We shall prove that $a_{1}+a_{2}-1 \leq p$. If $a_{2} \leq 2$ this is trivial: $a_{1}+a_{2}-1 \leq 3 \leq p$. Let $a_{2} \geq 3$. From $(25)$ we have $a_{i} \geq 3, i=2, \ldots, r$. From these inequalities and the statement of the theorem

$$
\sum_{i=1}^{r}\left(a_{i}-1\right)+1=p+2
$$

we have

$$
p+2 \geq 1+\left(a_{2}-1\right)+\left(a_{1}-1\right)+2(r-2) .
$$

From this inequality and $r \geq 3$ it follows that $a_{1}+a_{2}-1 \leq p$. Thus we can now use the inductive assumption and obtain

$$
\Gamma_{p} \rightarrow\left(a_{1}+a_{2}-1, a_{3}, \ldots, a_{r}\right)
$$

Consider an arbitrary $r$-coloring $V_{1} \cup \ldots \cup V_{r}$ of $V\left(\Gamma_{p}\right)$. Let us assume that $V_{i}$ does not contain an $a_{i}$-clique for each $i=3, \ldots, r$. Then from (26) we have $V_{1} \cup V_{2}$ contains $\left(a_{1}+a_{2}-1\right)$-clique. Now from the pigeonhole principle it follows that either $V_{1}$ contains an $a_{1}$-clique or $V_{2}$ contains an $a_{2}$-clique. This completes the proof of Theorem 1.

\section{Proof of the Main Theorem}

Let $m$ and $p$ be positive integers $p \geq 3$ and $m \geq p+2$. We shall first prove that for arbitrary positive integers $a_{1}, \ldots, a_{r}$ such that

$$
m=1+\sum_{i=1}^{r}\left(a_{i}-1\right)
$$

and $\max \left\{a_{1}, \ldots, a_{r}\right\} \leq p$ we have

$$
K_{m-p-2}+\Gamma_{p} \rightarrow\left(a_{1}, \ldots, a_{r}\right)
$$

We shall prove (27) by induction on $t=m-p-2$. As $m \geq p+2$ the base is $t=0$ and it follows from Theorem 1 . Assume now $t \geq 1$. Then obviously

$$
K_{m-p-2}+\Gamma_{p}=K_{1}+\left(K_{m-p-3}+\Gamma_{p}\right)
$$


Let $V\left(K_{1}\right)=\{w\}$. Consider an arbitrary $r$-coloring $V_{1} \cup \ldots \cup V_{r}$ of $V\left(K_{m-p-2}+\Gamma_{p}\right)$. Let $w \in V_{i}$ and $V_{j}, j \neq i$, does not contain an $a_{j}$-clique.

In order to prove (27) we need to prove that $V_{i}$ contains an $a_{i}$-clique. If $a_{i}=1$ this is clear as $w \in V_{i}$. Let $a_{i} \geq 2$. According to the inductive hypothesis we have

$$
K_{m-p-3}+\Gamma_{p} \rightarrow\left(a_{1}, \ldots, a_{i-1}, a_{i}-1, a_{i+1}, \ldots, a_{r}\right) .
$$

We consider the coloring

$$
V_{1} \cup \ldots \cup V_{i-1} \cup\left\{V_{i}-w\right\} \cup \ldots \cup V_{r}
$$

of $V\left(K_{m-p-3}+\Gamma_{p}\right)$. As $V_{j}, j \neq i$, do not contain $a_{j}$-cliques, from (28) we have that $V_{i}-\{w\}$ contains an $\left(a_{i}-1\right)$-clique. This $\left(a_{i}-1\right)$-clique together with $w$ form an $a_{i}$-clique in $V_{i}$. Thus (27) is proved.

From Corollary 1 obviously follows that $c l\left(K_{m-p-2}+\Gamma_{p}\right)=m-2$. From this and (27) we have $K_{m-p-2}+\Gamma_{p} \in H\left(a_{1}, \ldots, a_{r} ; m-1\right)$. The number of the vertices of the graph $K_{m-p-2}+\Gamma_{p}$ is $m+3 p$ therefore $F\left(a_{1}, \ldots, a_{r} ; m-1\right) \leq m+3 p$.

The main theorem is proved.

Acknowledgements. We are very grateful to the anonymous referee whose important recommendations improved the presentation a lot.

\section{References}

[1] J. Coles, Algorithms for bounding Folkman numbers. Master thesis, http://www.jpcoles.com/uni/rit/thesis/jpc-thesis-folkman.pdf.

[2] J. Coles, S. Radziszowski, Computing the Folkman number $F_{v}(2,2,3 ; 4)$, http://www.cs.rit.edu/ spr/PUBL/paper50.pdf, submitted.

[3] J. Folkman, Graphs with monochromatic complete subgraphs in every edge coloring, SIAM. J. Appl. Math. 18, 1970, 19-24.

[4] P. Guta. On the structure of k-chromatic critical graphs of order $k+p$, Stud. Cern. Math., 50, 1988, N 3-4, 169-173.

[5] T. Łuczak, S. Urbański, A note on restricted vertex Ramsey numbers, Period. Math. Hung., 33, 1996, 101-103.

[6] T. Łuczak, A. Ruciński, S. Urbański, On minimal vertex Folkman graphs, Discrete Math., 236, 2001, 245-262.

[7] N. Nenov, On the Zykov numbers and some its applications to Ramsey theory, Serdica Bulgaricae math. publicationes 9, 1983, 161-167 (in Russian).

[8] N. Nenov, On a class of vertex Folkman graphs, Annuaire Univ. Sofia Fac. Math. Inform. 94, 2000, 15-25.

[9] K. Piwakowski, S. Radziszowski, S. Urbański, Computation of the Folkman number $F_{e}(3,3 ; 5)$. J. Graph Theory 32, 1999, 41-49. 